\title{
THE SINGULAR POINTS OF ANALYTIC SPACE-CURVES*
}

\section{BY \\ ARTHUR RANUM}

1. The singular points of analytic space-curves have been investigated by various writers. $\dagger$ The object of this paper is to simplify the theory and also to make it self-dual by introducing the dual counterparts of the ordinary curvature and spherical curvature. Let

$$
x=x(t), \quad y=y(t), \quad z=z(t)
$$

be the equations of a twisted curve, the functions being analytic. Then in the neighborhood of any given point $P_{0}$ of the curve, at which $t=t_{0}$, there exists a certain region of the complex $t$-plane, within which $x, y, z$ are expressible as series of powers of $t-t_{0}$ (with positive integral exponents), and such that to different values of $t$ in the region will correspond different points.

The point $P_{0}$ will be singular, in the broader sense which we shall adopt, $\ddagger$ if

$$
\left|\begin{array}{ccc}
x^{\prime} & y^{\prime} & z^{\prime} \\
x^{\prime \prime} & y^{\prime \prime} & z^{\prime \prime} \\
x^{\prime \prime \prime} & y^{\prime \prime \prime} & z^{\prime \prime \prime}
\end{array}\right|=0,
$$

when $t=t_{0}$; here $x^{\prime}=d x / d t$, etc. The points for which $x^{\prime}=y^{\prime}=z^{\prime}=0$, to which the term singular is sometimes confined, are therefore only a special case.

2. With every point $P_{0}$, singular or non-singular, there is associated a triad of positive integers $(\alpha, \beta, \gamma)$, such that if we put

* Presented, in part, to the American Mathematical Society, December 27, 1915. Abstract in the Bulletin of the American Mathematical Society, vol. 22 (1916), pp. 265, 268.

$\dagger$ Fine, American Journal of Mathematics, vol. 8 (1886), pp. 156-177; Björling, Archiv der Mathematik und Physik, (2), vol. 8 (1890), pp. 83-91; Wölffing, Archiv der Mathematik und Physik, (2), vol. 15 (1897), pp. 146-158; Burali-Forti, Atti di Torino, vol. 36 (1901), pp. 935-938; Mehmke, Zeitschrift für Mathematik und Physik, vol. 49 (1903), pp. 62-83; Saurel, Annals of Mathematics, (2), vol. 7 (1905), pp. 3-9; von Lilienthal, Vorlesungen über Differentialgeometrie, vol. 1, Leipzig, 1908, pp. 242-262; Meder, Crelle's Journal, vol. 137 (1910), pp. 83-144; Zindler, Wiener Sitzungsberichte, vol. $127 \mathrm{I}$ (1918), pp. 871-918; Study, Mathematische Annalen, vol. 87 (1922), pp. 207-228.

$\ddagger$ The definitions used by Lilienthal (loc. cit., p. 182), Study (p. 212), Burali-Forti (p. 935) and Mehmke (p. 71) are all practically the same as this; but no writer, so far as I am aware, writes the condition in this simple form. Lilienthal gives three possibilities, but the first two are included in the third. 


$$
\Delta=\Delta(\alpha, \beta, \gamma)=\left|\begin{array}{lll}
x^{(\alpha)} & y^{(\alpha)} & z^{(\alpha)} \\
x^{(\alpha+\beta)} & y^{(\alpha+\beta)} & z^{(\alpha+\beta)} \\
x^{(\alpha+\beta+\gamma)} & y^{(\alpha+\beta+\gamma)} & z^{(\alpha+\beta+\gamma)}
\end{array}\right|
$$

then

$$
\Delta \neq 0 \text {, when } t=t_{0},
$$

whereas every determinant $\Delta\left(\alpha^{\prime}, \beta^{\prime}, \gamma^{\prime}\right)$ vanishes, if the triad $\left(\alpha^{\prime}, \beta^{\prime}, \gamma^{\prime}\right)$ precedes $(\alpha, \beta, \gamma)$ lexicographically, which means that either

or

$$
\alpha^{\prime}<\alpha,
$$

or

$$
\alpha^{\prime}=\alpha, \quad \beta^{\prime}<\beta,
$$

$$
\alpha^{\prime}=\alpha, \quad \beta^{\prime}=\beta, \quad \gamma^{\prime}<\gamma^{*}
$$

Hence $P_{0}$ is a non-singular point, if $\alpha=\beta=\gamma=1$, and is singular, if $\alpha+\beta+\gamma$ $>3$. We shall call $(\alpha, \beta, \gamma)$ the type $\dagger$ of the point $P_{0}$ or of the curve (namely the region in the neighborhood of $\left.P_{0}\right)$, writing $P_{0}=(\alpha, \beta, \gamma)$.

By the use of homogeneous coördinates it has been shown that the type of a curve is invariant under the group of collineations, and that every correlation transforms a curve of type $(\alpha, \beta, \gamma)$ into one of type $(\gamma, \beta, \alpha)$, so that these two types are dual. Hence the type of a curve is a concept belonging to projective, as well as to metric, differential geometry.

We define $w$ and $W$ by the equations

$$
\begin{aligned}
w & =\left(\sum\left(x^{(\alpha)}\right)^{2}\right)^{1 / 2}, \\
W & =\left(\sum\left(y^{(\alpha)} z^{(\alpha+\beta)}-z^{(\alpha)} y^{(\alpha+\beta)}\right)^{2}\right)^{1 / 2},
\end{aligned}
$$

where the summations are cyclic with respect to $x, y, z$, and where $t$ is put equal to $t_{0}$.

3. From now on we shall assume that $t$ is real and that $x, y, z$ are real functions of $t$. Then the conditicn $\left(3^{\prime}\right)$ shows that $w W \neq 0$; and by choosing the positive square roots we have $w>0, W>0$.

If we substitute a new parameter $t$ for $t-t_{0}$ and also choose the coördinate axes so that the origin $O$ coincides with $P_{0}$, the $x$-axis with the tangent at $P_{0}$, and the $x y$-plane with the osculator (osculating plane) at $P_{0}$, then the equations of the curve (in the given region or interval) become

* See Lilienthal, pp. 242-251; Mehmke, pp. 68-70; Study, p. 210.

$\dagger$ Lilienthal appears to be the only writer who uses the integers $\alpha, \beta, \gamma$, which he calls $\nu_{1}, \nu_{2}, \nu_{2}$; Study calls the triad $(\alpha-1, \beta-1, \gamma-1)$ the characteristic of the point $P_{0}$, writing it $\left(k_{1}, k_{2}, k_{3}\right)$.

† Björling, p. 85; Study, p. 212. 


$$
\begin{aligned}
& x=a t^{\alpha}+\cdots, \\
& y=b t^{\alpha+\beta}+\cdots, \\
& z=c t^{\alpha+\beta+\gamma}+\cdots,
\end{aligned}
$$

where $a b c \neq 0$ and we can choose $a>0, b>0, c>0$. Then

(6) $w=\alpha ! a$,

$$
W=\alpha !(\alpha+\beta) ! a b, \quad|\Delta|=\alpha !(\alpha+\beta) !(\alpha+\beta+\gamma) ! a b c,
$$

and therefore

$$
a=\frac{w}{\alpha !}, \quad b=\frac{W}{w(\alpha+\beta) !}, \quad c=\frac{|\Delta|}{W(\alpha+\beta+\gamma) !} .
$$

The exponents of $t$ in the terms actually present in the expansions (5) will have no common factor; but of course $\alpha, \beta, \gamma$ may have a common factor.

The positive branch of the curve, corresponding to small positive values of $t$, will lie in the octant $[+1,+1,+1]$, in which $x, y, z$ are all positive; and the negative branch will lie in the octant $\left[(-1)^{\alpha},(-1)^{\alpha+\beta},(-1)^{\alpha+\beta+\gamma}\right]$, which is therefore determined by the evenness or oddness of $\alpha, \beta$, and $\gamma$. This gives the first rough classification of singular points into eight categories.

We also arrive at the same classification in a more significant, because self-dual, manner by considering the curve to be generated by three elements, namely a point, a line (the tangent), and a plane (the osculator), and allowing each of these elements to be either ordinary or a reversal-element (Rückkehrelement).

It is known* that as $t$ decreases (or increases) through the value zero, then at the origin $O$ :

the generating point continues or the generating osculator continues or reverses the direction of its motion reverses the direction of its rotation along the tangent, according as $\alpha$ is about the tangent, according as $\gamma$ is odd or even; odd or even;

and the generating tangent continues or reverses the direction of its rotation in the osculator (or about the point), according as $\beta$ is odd or even.

4. These results are expressed in the following table. In each case, as $t$ decreases through the value zero, the generating point emerges from the first octant $[+1,+1,+1]$ into the octant indicated, whose number, given in the last column, is also the number of the category to which the curve (or point) belongs. The octants 1, 2, 3, 4, for which $z>0$, are numbered like the corresponding quadrants in the $x y$-plane, and the octants $5,6,7,8$ are

* Fine, pp. 166, 173; Saurel, pp. 6-7. 
symmetric to the respective octants $1,2,3,4$ with respect to the origin, and not with respect to the $x y$-plane, as Lilienthal (p. 202) numbers them.

\begin{tabular}{|c|c|c|c|c|c|c|}
\hline$\alpha$ & $\begin{array}{c}\text { Type } \\
\beta\end{array}$ & $\boldsymbol{\gamma}$ & $(-1)^{\alpha}$ & $\begin{array}{l}\text { Octant }( \\
(-1)^{\alpha+\beta}\end{array}$ & $\begin{array}{l}(-1)^{\alpha+\beta+\gamma} \\
0\end{array}$ & $\begin{array}{l}\text { Number of Octant } \\
\text { and of Category }\end{array}$ \\
\hline even & even & even & +1 & +1 & +1 & 1 \\
\hline $\begin{array}{l}\text { odd } \\
\text { odd } \\
\text { even }\end{array}$ & $\begin{array}{l}\text { odd } \\
\text { even } \\
\text { odd }\end{array}$ & $\begin{array}{l}\text { even } \\
\text { odd } \\
\text { odd }\end{array}$ & $\begin{array}{l}-1 \\
-1 \\
+1\end{array}$ & $\begin{array}{l}+1 \\
-1, \\
-1\end{array}$ & $\begin{array}{l}+1 \\
+1 \\
+1\end{array}$ & $\begin{array}{l}2 \\
3 \\
4\end{array}$ \\
\hline $\begin{array}{l}\text { odd } \\
\text { even } \\
\text { even }\end{array}$ & $\begin{array}{l}\text { even } \\
\text { odd } \\
\text { even }\end{array}$ & $\begin{array}{l}\text { even } \\
\text { even } \\
\text { odd }\end{array}$ & $\begin{array}{l}-1 \\
+1 \\
+1\end{array}$ & $\begin{array}{l}-1 \\
-1 \\
+1\end{array}$ & $\begin{array}{l}-1 \\
-1 \\
-1\end{array}$ & $\begin{array}{l}5 \\
6 \\
7\end{array}$ \\
\hline odd & odd & odd & -1 & +1 & -1 & 8 \\
\hline
\end{tabular}

In the case of a singular point of the $2 \mathrm{~d}, 3 \mathrm{~d}$, or 4 th category, one of the three generating elements is a reversal-element and the other two are ordinary; the $2 \mathrm{~d}$ and 4 th categories are dual and the $3 \mathrm{~d}$ is self-dual. Similarly the 5 th and 7 th are dual and the 6 th is self-dual. The 1 st and 8 th are also self-dual; the 8 th includes the non-singular points $(1,1,1){ }^{*}$

The positive and negative branches of the curve will lie on the same side of the osculating plane (categories 1, 2, 3, 4) or on opposite sides (categories $5,6,7,8)$, according as the number of reversal-elements is odd or even.

5. The interval $(-h<t<h)$ within which the curve is defined by (5) can be chosen such that every point except the origin $O$ is non-singular. At any such point $P=(x, y, z)$ consider the six variables

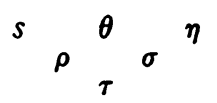

where $1 / \rho$ and $1 / \tau$ are the ordinary curvature and torsion and where

$$
s=\int_{0}^{t}\left(\sum x^{\prime 2}\right)^{1 / 2} d t, \quad \theta=\int_{0}^{t} d s / \rho, \quad \eta=\int_{0}^{t} d s / \tau \text { and } \sigma=\rho / \tau .
$$

Then $\rho=d s / d \theta, \tau=d s / d \eta$ and $\sigma=d \eta / d \theta ; d s, d \theta$ and $d \eta$ are the element of arc,

- Mehmke (pp. 65-67) and Zindler (pp. 876-879) give distinctive names to these eight categories; Zindler's names seem to be highly appropriate, except that in accordance with his definition a point of the 8th category is called non-singular (gewöhnlich); it would be better to call it a "Gegenhenkel." 
the angle of contingence and the angle of torsion, respectively; $s$ is dual to $\eta$, $\rho$ to $\sigma$, and $\theta, \tau$ are self-dual. Following Plucker* we shall call $\sigma$ the conical curvature at $P$.

We define the value of $\rho$ at a singular point $O$ to be the limit of its value at $P$ as $t \rightarrow 0$, including the case where $\rho \rightarrow \infty$; similarly for $\tau$ and $\sigma$.

6. We now proceed to find a second classification of the singular points $O$ into thirteen classes, depending on whether the values of $\rho, \tau$ and $\sigma$ at $O$ are zero, finite and different from zero, or infinite. Since $\sigma=-\cot \omega$, where $\omega$ is the angle between the tangent and the rectifying line, $\sigma$ will be zero, infinite, or neither, according as the rectifying line coincides with the binormal, with the tangent, or with neither one.

From (5) by means of known formulas we easily derive for the values of $\rho, \tau$ and $\sigma$ at the non-singular point $P$ the following expansions in ascending integral powers of $t$, in which the exponents of $t$ in the initial terms are sometimes negative:

$$
\begin{aligned}
& \rho=\frac{\alpha^{2} a^{2}}{\beta(\alpha+\beta) b} t^{\alpha-\beta}+\cdots, \\
& \tau=-\frac{\alpha \beta(\alpha+\beta) a b}{\gamma(\beta+\gamma)(\alpha+\beta+\gamma) c} t^{\alpha-\gamma}+\cdots, \\
& \sigma=-\frac{\alpha \gamma(\beta+\gamma)(\alpha+\beta+\gamma) a c}{\beta^{2}(\alpha+\beta)^{2} b^{2}} t^{\gamma-\beta}+\cdots .
\end{aligned}
$$

We also find that

and therefore that

$$
\begin{aligned}
& s^{\prime}=\alpha a t^{\alpha-1}+\cdots \\
& \theta^{\prime}=\frac{\beta(\alpha+\beta) b}{\alpha a} t^{\beta-1}+\cdots, \\
& \eta^{\prime}=-\frac{\gamma(\beta+\gamma)(\alpha+\beta+\gamma) c}{\beta(\alpha+\beta) b} t^{\gamma-1}+\cdots,
\end{aligned}
$$

$$
\begin{aligned}
& s=a t^{\alpha}+\cdots, \\
& \theta=\frac{(\alpha+\beta) b}{\alpha a} t^{\beta}+\cdots, \\
& \eta=-\frac{(\beta+\gamma)(\alpha+\beta+\gamma) c}{\beta(\alpha+\beta) b} t+\cdots .
\end{aligned}
$$

- Geometrie des Raumes, 1846, pp. 34-35. See also E. Müller, Wiener Sitzungsberichte, vol. 126 (1917), pp. 311-312. In the Quarterly Journal of Mathematics, vol. 46 (1915), p. 36, I called $\sigma\left(=1 / r^{\prime}\right)$ the plane-curvature. 
The leading coefficient can never be zero in any of these nine expansions. The leading terms in $\left(8_{1}\right),\left(8_{2}\right)$ and $\left(8_{3}\right)$, under the respective hypotheses $\alpha=\beta, \alpha=\gamma$ and $\beta=\gamma$, become equal to $l, m$ and $n$, respectively, where

$$
\begin{gathered}
l=\frac{a^{2}}{2 b}, m=-\frac{\beta a b}{(2 \alpha+\beta) c}, \\
n=-\frac{2 \alpha(\alpha+2 \beta) a c}{(\alpha+\beta)^{2} b^{2}} .
\end{gathered}
$$

If $\alpha=\beta=\gamma$, then $m$ and $n$ become simpler in form and we have

$$
l=a^{2} /(2 b), \quad m=-a b /(3 c), \quad n=-3 a c /\left(2 b^{2}\right) .
$$

We now pass to the limit by letting $t \rightarrow 0$ and obtain for the values of $\rho, \tau$ and $\sigma$ at the origin $O$ the following:

$$
\begin{aligned}
& \rho=\left\{\begin{array}{c}
0 \\
l \\
\infty
\end{array}\right\} \text { according as } \alpha\left\{\begin{array}{l}
\geqq \\
\overline{<}
\end{array}\right\} \beta, \\
& \tau=\left\{\begin{array}{c}
0 \\
m \\
\infty
\end{array}\right\} \text { according as } \alpha\left\{\begin{array}{l}
\geqq \\
\overline{<}
\end{array}\right\} \gamma, \\
& \sigma=\left\{\begin{array}{c}
0 \\
n \\
\infty
\end{array}\right\} \text { according as } \gamma\left\{\begin{array}{l}
\geqq \\
\overline{<}
\end{array}\right\} \beta,
\end{aligned}
$$

where $l, m$ and $n$, given by (11), are finite and $\neq 0$. By means of (7), (3) and (4) we can, if we wish, express $l, m$ and $n$ in terms of the original equation (1) of the curve.

By examining $\left(12_{2}\right)$ and $\left(11_{2}\right)$ we see that if a point $O$ is of type $(\alpha, \beta, \alpha)$, then the absolute value of $\tau$ at $O$ is always less than $a b / c$, and is greater than, equal to, or less than $a b /(3 c)$, according as $\beta$ is greater than, equal to, or less than $\alpha$; also if $\alpha$ is fixed and $\beta \rightarrow \infty$, then $|\tau| \rightarrow a b / c$, and if $\beta$ is fixed and $\alpha \rightarrow \infty$, then $|\tau| \rightarrow 0$.

By a similar examination of $\left(12_{3}\right)$ and $\left(11_{3}\right)$ we see that if a point is of type $(\alpha, \beta, \beta)$, then $|\sigma|$ is always less than $2 a c / b^{2}$, and is $>,=$, or $\left\langle 3 a c /\left(2 b^{2}\right)\right.$, according as $\beta$ is $<,=$, or $>\alpha$. Hence $\omega$ (an acute angle) is always greater than $\cot ^{-1}\left(2 a c / b^{2}\right)$, and is $<,=$, or $>\cot ^{-1}\left(3 a c /\left(2 b^{2}\right)\right)$, according as $\beta$ is $\left\langle,=\right.$, or $>\alpha$; also if $\alpha \rightarrow \infty$, then $\omega \rightarrow \cot ^{-1}\left(2 a c / b^{2}\right)$, and if $\beta \rightarrow \infty$, then $\omega \rightarrow \pi / 2$.

7. Formulas (12) show us that the class to which any real and finite point of type $(\alpha, \beta, \gamma)$ belongs depends only on the relative magnitudes of $\alpha, \beta$, and $\gamma$. From this fact or the relation $\rho=\sigma \tau$ we see that the number of classes, instead of being $3^{3}$, reduces to 13 , as is shown in the following table: 


\begin{tabular}{c|c|c|c|c}
\hline Class-Number & Type & $\rho$ & $\tau$ & $\sigma$ \\
\hline 1 & $\alpha=\beta=\gamma$ & $\frac{a^{2}}{2 b}$ & $-\frac{a b}{3 c}$ & $-\frac{3 a c}{2 b^{2}}$ \\
\hline 2 & $\alpha=\gamma<\beta$ & $\infty$ & $m$ & $\infty$ \\
3 & $\alpha=\gamma>\beta$ & 0 & $m$ & 0 \\
\hline 4 & $\alpha=\beta<\gamma$ & $l$ & $\infty$ & 0 \\
5 & $\gamma=\beta<\alpha$ & 0 & 0 & $n$ \\
\hline 6 & $\alpha=\beta>\gamma$ & $l$ & 0 & $\infty$ \\
7 & $\gamma=\beta>\alpha$ & $\infty$ & $\infty$ & $n$ \\
\hline 8 & $\alpha<\beta<\gamma$ & $\infty$ & $\infty$ & 0 \\
9 & $\gamma<\beta<\alpha$ & 0 & 0 & $\infty$ \\
\hline 10 & $\alpha<\gamma<\beta$ & $\infty$ & $\infty$ & $\infty$ \\
11 & $\gamma<\alpha<\beta$ & $\infty$ & 0 & $\infty$ \\
\hline 12 & $\beta<\alpha<\gamma$ & 0 & $\infty$ & 0 \\
13 & $\beta<\gamma<\alpha$ & 0 & 0 & 0 \\
\hline
\end{tabular}

In each of the six classes 2-7 exactly two of the type-integers $\alpha, \beta, \gamma$ are equal and therefore exactly one of the variables $\rho, \tau, \sigma$ is finite and $\neq 0$. In each of the last six classes 8-13 the three integers are all distinct and $\rho, \tau, \sigma$ are all $=0$ or $\infty$. Classes 1,2 and 3 , in which $\tau$ is finite and $\neq 0$, are self-dual; and 4 is dual to 5,6 to 7,8 to 9,10 to 11 , and 12 to 13 . In any pair of dual classes the values of $\rho$ and $\sigma$ in one are interchanged in the other (except for the difference between $l$ and $n$ ).

8. From our cross-classification of singular points into eight categories and thirteen classes, by combination, we obtain a classification into species; the species of a point depends, therefore, on the evenness or oddness of $\alpha, \beta$, $\gamma$ and also on their relative magnitudes. Since these two properties are not entirely independent, the number of species is less than $8 \cdot 13=104$.

First, a singular point of class 1 must clearly be of category 1 or 8 . Hence if $\rho, \tau$ and $\sigma$ are all finite and different from zero, the curve must either emerge (for $t<0)$ in the 8 th octant or return to the 1 st octant. This gives two species.

Next, in each of the six classes 2-7 two of the integers $\alpha, \beta, \gamma$, being equal, must be either both odd or both even, while the third may be odd or even. This gives $6 \cdot 4=24$ species.

In particular, putting $\alpha=\beta(\rho=l)$, we see that the only categories of singular points at which the ordinary curvature $1 / \rho$ can be finite and different from zero are the $1 s t, 8 t h, 2 d$ and 7 th, for which the negative branch of the curve lies in one of the octants $\left[(-1)^{\alpha},+1,(-1)^{\gamma}\right]$.

Similarly $(\alpha=\gamma, \tau=m)$ the only categories of points at which the torsion $1 / \tau$ can be finite and different from zero are the 1 st, 8 th, $3 d$ and 6 th, for which 
the negative branch of the curve lies in one of the octants $\left[(-1)^{\alpha},(-1)^{\alpha+\beta}\right.$, $\left.(-1)^{\beta}\right]$. Of these four octants no two are adjacent (symmetric with respect to a plane).

Again $(\beta=\gamma, \sigma=n)$ the only categories of points at which the conical curvature $\sigma$ can be finite and different from zero are the 1 st, 8 th, 4 th, and 5 th, for which the negative branch of the curve lies in one of the octants $\left[(-1)^{\alpha},(-1)^{\alpha+\beta},(-1)^{\alpha}\right]^{*}$

In other words, if the negative branch of the curve lies in the $2 d$ or 7 th octant, $\tau$ and $\sigma$ must be zero or infinite; if in the $3 d$ or 6 th octant, $\rho$ and $\sigma$ must be zero or infinite; if in the 4th or 5 th octant, $\rho$ and $\tau$ must be zero or infinite.

Finally, in each of the six classes 8-13 the integers $\alpha, \beta, \gamma$ are unrestricted as to oddness or evenness; that is, a point at which $\rho, \tau, \sigma$ are all zero or infinite may belong to any one of the eight categories. This gives $6 \cdot 8=48$ species. The total number of species is therefore $2+24+48=74$. $\dagger$

9. Since every point belonging to a self-dual class $(\alpha=\gamma, \tau=m)$ will also belong to a self-dual category, the self-dual species are precisely those that belong to the classes $1,2,3$, and their number is $2+4+4=10$.

It is evident that the species of a point is invariant under all the real collineations that carry the point into a finite point.

If the curve is imaginary, our entire classification falls to the ground, classes as well as categories; for by (4), (7) and (11) we see that $w$ and $W$ may vanish and that $a, b, c, l, m, n$ may not be determined. For instance, the origin is a non-singular point $(1,1,1)$ of the curve $x=i t, y=t+t^{2}, z=t^{3}$, and yet since $w=0, W=2 i$ and $\Delta=12 i$, we have $\rho=\sigma=0, \tau=i / 3$, which contradicts (12).

The simplest singular points are $(2,1,1),(1,2,1)$ and $(1,1,2)$; the first is said to be a stationary point (of the first degree), at the second the tangent is said to be stationary, and at the third the osculator is said to be stationary. Fine (p. 174) has shown that if the curve is algebraic, then with respect to its effect on the order, class, rank, and genus of the curve, a singular point of type $(\alpha, \beta, \gamma)$ is equivalent to $\alpha-1$ stationary points, $\beta-1$ stationary tangents and $\gamma-1$ stationary osculators.

Study (p. 214) has also shown that $(\alpha, \beta, \gamma)$ is an $\alpha$-fold point of the curve, the corresponding tangent is $\beta$-fold. and the osculator is $\gamma$-fold.

* The first two parts of this triple theorem were proved by Mehmke (pp. 75, 77).

$\dagger$ Burali-Forti (p. 938) and Mehmke (p. 78), by ignoring $\sigma$, obtain a classification that is not self-dual. Instead of 13 classes and 74 species they obtain only 9 classes and 50 species. For them our classes 5,9 and 13 coincide; also 7,8 and 10 . For them the points $(1,2,4),(1,2,2)$ and $(1,4,2)$ are of the same species, although the corresponding values of $\sigma$ are $0, n$ and $\infty$, respectively.

$\ddagger$ Meder (loc. cit.) studies the "Ordnungszahlen" of $\rho, \tau$ and various other variables at an infinite point. 


\section{Projections on the coördinate planes}

10. Now consider the orthogonal projections of a curve $C$ on the osculating, rectifying and normal planes at a point $O=(\alpha, \beta, \gamma)$. From (5) we see that the types of these three plane curves at their common point are

$$
\begin{aligned}
& O_{x y}=(\alpha, \beta), \\
& O_{x z}=(\alpha, \beta+\gamma), \\
& O_{y z}=(\alpha+\beta, \gamma),
\end{aligned}
$$

respectively. Also consider a curve $C^{\prime}$ dual to $C$ and on it the point $O^{\prime}$ that corresponds to $O$ in a correlation; then $O^{\prime}=(\gamma, \beta, \alpha), O_{x z}^{\prime}=(\gamma, \alpha+\beta)$ and $O_{y z}^{\prime}=(\beta+\gamma, \alpha)$. But in plane geometry a point $(\lambda, \mu)$ is dual to a point $(\mu, \lambda)$. Hence $O_{y z}^{\prime}$ is dual to $O_{x z}$ and $O_{x z}^{\prime}$ to $O_{y z}$. That is, if two space-curves are dual, the projection of either on the normal plane at a point $O$ is dual (planar duality) to the projection of the other on the rectifying plane at the corresponding point $O^{\prime}$.

If $\alpha=\beta+\gamma, O_{x z}$ (and therefore also $O_{y z}^{\prime}$ ) is self-dual, and conversely. If $\alpha+\beta=\gamma, O_{y z}$ (and therefore also $O_{x z}^{\prime}$ ) is self-dual, and conversely.

If $\alpha=\gamma$, so that $O$ is self-dual (spatial duality), then $O_{x z}=(\alpha, \alpha+\beta)$ and $O_{y z}=(\alpha+\beta, \alpha)$, which are therefore dual (planar duality), and conversely. That is, if the torsion at a point $O$ is finite and different from zero, the projections of the curve on the normal and rectifying planes at $O$ are dual, and conversely.

11. It is known* that the singular points of plane curves are divided into four categories (quadrants), three classes $(\rho=0, \infty$, or neither) and ten species. Consider the possible combinations of categories (classes, species) of the three projections (13). The combinations of categories are obviously eight in number, and there is a one-to-one correspondence between the eight octants in which the negative branch of the curve $C$ may lie and the eight combinations of quadrants in which the negative branches of the projections may lie. $\dagger$

12. When we come to the combinations of classes or species, we find a very different situation. Let $\rho_{x y}, \rho_{x z}$ and $\rho_{y z}$ be the respective radii of curvature of the three projections (13) at their common point. Of course $\rho_{x y}=\rho$, and its value is determined by $\left(12_{1}\right)$ and $\left(11_{1}\right)$. Similarly $\rho_{x z}$ and $\rho_{y z}$ are determined as follows:

* See Burali-Forti, p. 938.

† See the figures drawn by Mehmke, pp. 65-67. 
where

$$
\begin{aligned}
& \rho_{x z}=\left\{\begin{array}{c}
0 \\
k_{1} \\
\infty
\end{array}\right\}, \text { according as } \alpha\left\{\begin{array}{l}
\geq \\
\equiv \\
<
\end{array}\right\} \beta+\gamma, \\
& \rho_{y z}=\left\{\begin{array}{c}
0 \\
k_{2} \\
\infty
\end{array}\right\}, \text { according as } \alpha+\beta\left\{\begin{array}{l}
\geq \\
\overline{<}
\end{array}\right\} \gamma,
\end{aligned}
$$

$$
k_{1}=a^{2} /(2 c) \neq 0, \quad k_{2}=b^{2} /(2 c) \neq 0 .
$$

The number of combinations of classes is only eleven. For if $\alpha \leqq \beta$, then $\alpha<\beta+\gamma$ and $\gamma$ may be either $<,=$, or $>\alpha+\beta$; this gives $2 \cdot 3=6$ combinations. But if $\alpha>\beta, \gamma$ may be either $\geqq \alpha+\beta$ or $\leqq \alpha-\beta$, or finally we may

\begin{tabular}{|c|c|c|c|c|c|c|}
\hline & & Type & & $\rho_{x y}(=\rho)$ & $\rho_{x s}$ & $\rho_{y z}$ \\
\hline 1 & $\alpha<\beta$ & $\alpha<\beta+\gamma$ & $\alpha+\beta<\gamma$ & $\infty$ & $\infty$ & $\infty$ \\
\hline 2 & a & « & $\alpha+\beta=\gamma$ & $\infty$ & $\infty$ & $k_{2}$ \\
\hline 3 & « & “ & $\alpha+\beta>\gamma$ & $\infty$ & $\infty$ & 0 \\
\hline 4 & $\alpha=\beta$ & “ & $\alpha+\beta<\gamma$ & $l$ & $\infty$ & $\infty$ \\
\hline 5 & “ & “ & $\alpha+\beta=\gamma$ & $l$ & $\infty$ & $k_{2}$ \\
\hline 6 & “ & « & $\alpha+\beta>\gamma$ & $l$ & $\infty$ & 0 \\
\hline 7 & $\alpha>\beta$ & “ & $\alpha+\beta<\gamma$ & 0 & $\infty$ & $\infty$ \\
\hline 8 & « & “ & $\alpha+\beta=\gamma$ & 0 & $\infty$ & $k_{2}$ \\
\hline 9 & a & “ & $\alpha+\beta>\gamma$ & 0 & $\infty$ & 0 \\
\hline 10 & “ & $\alpha=\beta+\gamma$ & a & 0 & $k_{1}$ & 0 \\
\hline 11 & “ & $\alpha>\beta+\gamma$ & “ & 0 & 0 & 0 \\
\hline
\end{tabular}
have $\alpha+\beta>\gamma>\alpha-\beta$; this gives 5 combinations. Altogether $6+5=11$ combinations, which are exhibited in the following table.

If $\alpha \geqq \beta+\gamma$, then $\alpha>\beta$ and $\alpha+\beta>\gamma$. Hence by (14) and (121) or by an inspection of the table, we see that the only points $O$ for which the radius of curvature $\rho_{x s}$ of the projection of the curve on the rectifying plane at $O$ is not infinite are those for which the radii of curvature $\rho_{x y}$ and $\rho_{y z}$ of the projections on the osculating and normal planes are both zero.

Putting $\alpha=\beta+\gamma$ or $\alpha+\beta=\gamma$, we easily find, by $\$ 4$ or by a known theorem on plane curves, that if the two branches of the curve at a point $O$ lie on opposite sides of the osculating plane, then the radii of curvature $\rho_{x z}$ and $\rho_{y z}$ of its projections on the rectifying and normal planes must be zero or infinite.

13. The number of combinations of species is easily seen to be only 62, although each projection by itself is entirely unrestricted in species (ten cases). There is obviously no one-to-one correspondence between the 13 classes (74 species) of curves and the 11 combinations of classes (62 combinations of species) of their projections. Not only may two curves of different 
species have projections belonging to the same combination of species, but two curves of the same species may have projections belonging to different combinations of species (their projections $O_{x y}$ on the osculator must, of course, be of the same species).

The first possibility is illustrated by the types $(4,2,3)$ and $(4,2,5)$, for which $\tau=0$ and $\infty$, respectively, whereas their projections are of types $(4,2),(4,5),(6,3)$ and $(4,2),(4,7),(6,5)$, respectively, for which in both cases $\rho_{x y}=0, \rho_{x z}=\infty, \rho_{y z}=0$. The second possibility is illustrated by $(4,2,3)$, just considered, and $(6,2,3)$; these two types are clearly of the same species, whereas the projections of the latter type of curve are of types $(6,2),(6,5)$, $(8,3)$, for which $\rho_{x y}=\rho_{x z}=\rho_{y z}=0$.

In spite of this lack of uniformity in the correspondence, there is, of course, a close connection between the values of $\rho, \tau, \sigma$ and the values of $\rho_{x y}(=\rho)$, $\rho_{x z}, \rho_{y z}$. For instance:

If $\alpha \geqq \beta+\gamma$, then $\alpha>\beta$ and $\alpha>\gamma$. By (14) and (12) this says that if $\rho_{x z}=k_{1}$ or 0 , then $\rho=\tau=0$.

Hence, for every point $O$ except those at which $\rho=\tau=0$, the projection of the curve on the rectifying plane at $O$ has an infinite radius of curvature $\rho_{x z}$.
If $\gamma \geqq \beta+\alpha$, then $\gamma>\beta$ and $\gamma>\alpha$. By (14) and (12) this says that if $\rho_{y z}=k_{2}$ or $\infty$, then $\sigma=0$ and $\tau=\infty$.

Hence, for every point $O$ except those at which $\sigma=0$ and $\tau=\infty$, the projection of the curve on the normal plane at $O$ has a vanishing radius of curvature $\rho_{y z}$.

\section{SECTIONS OF THE TANGENT SURFACE BY THE CoÖRdinate PLANES}

14. The osculating, rectifying and normal planes at a point $O=(\alpha, \beta, \gamma)$ will cut the tangent surface of the curve in three plane curves $\bar{O}_{x y}, \bar{O}_{x z}$ and $\bar{O}_{y z}$, whose character at their common point $O$ we shall now consider. The parametric equations of the tangent surface of the curve (5), $\S 3$, are

$$
\begin{aligned}
& x=\left(a t^{\alpha}+\cdots\right)+u\left(\alpha a t^{\alpha-1}+\cdots\right), \\
& y=\left(b t^{\alpha+\beta}+\cdots\right)+u\left[(\alpha+\beta) b t^{\alpha+\beta-1}+\cdots\right], \\
& z=\left(c t^{\alpha+\beta+\gamma}+\cdots\right)+u\left[(\alpha+\beta+\gamma) c t^{\alpha+\beta+\gamma-1}+\cdots\right],
\end{aligned}
$$

where $t$ and $u$ are the parameters. The osculator $z=0$ will intersect the surface in points for which

$$
u=-\frac{1}{\alpha+\beta+\gamma} t+\cdots .
$$

Substituting this expansion of $u$ in $\left(16_{1}\right)$ and $\left(16_{2}\right)$, we find the equations of the section $\bar{O}_{x y}$ to be 


$$
x=\frac{\beta+\gamma}{\alpha+\beta+\gamma} a t^{\alpha}+\cdots, \quad y=\frac{\gamma}{\alpha+\beta+\gamma} b t^{\alpha+\beta}+\cdots .
$$

Similarly the equations of $\bar{O}_{x z}$ are found to be

$$
x=\frac{\beta}{\alpha+\beta} a t^{\alpha}+\cdots, \quad z=-\frac{\gamma}{\alpha+\beta} c t^{\alpha+\beta+\gamma}+\cdots,
$$

and the equations of $\bar{O}_{y z}$ to be

$$
y=-\frac{\beta}{\alpha} b t^{\alpha+\beta}+\cdots, \quad z=-\frac{\beta+\gamma}{\alpha} c t^{\alpha+\beta+\gamma}+\cdots .
$$

Since the leading coefficients in the six expansions of (17), (18) and (19) are all $\neq 0$, the sections $\bar{O}_{x y}, \bar{O}_{x z}$ and $\bar{O}_{y z}$ are of the same types as the respective projections $O_{x y}, O_{x z}$ and $O_{y z}$, as given by (13), $\$ 10$. Hence everything stated in $\$ \$ 10-13$ about the categories, classes and species of the three projections applies equally well to the three sections.

15. Let $\bar{p}_{x y}, \bar{p}_{x z}$ and $\bar{p}_{y z}$ be the respective radii of curvature of the three sections at the point $O$. Their values are easily found to be the following:

$$
\bar{p}_{x y}=\left\{\begin{array}{c}
0 \\
a_{0} \\
\infty
\end{array}\right\} \text { according as } \alpha\left\{\begin{array}{l}
\geq \\
<
\end{array}\right\} \beta,
$$

where

$$
a_{0}=\frac{(\alpha+\gamma)^{2} a^{2}}{2 \gamma(2 \alpha+\gamma) b}
$$

where

$$
\bar{\rho}_{x z}=\left\{\begin{array}{c}
0 \\
a_{1} \\
\infty
\end{array}\right\} \text { according as } \alpha\left\{\begin{array}{l}
\geq \\
\overline{<}
\end{array}\right\} \beta+\gamma
$$

where

$$
\begin{aligned}
a_{1} & =-\frac{\beta^{2} a^{2}}{2 \gamma(2 \beta+\gamma) c} ; \\
\bar{\rho}_{y z} & =\left\{\begin{array}{c}
0 \\
a_{2} \\
\infty
\end{array}\right\} \text { according as } \alpha+\beta\left\{\begin{array}{l}
\geqq \\
\equiv
\end{array}\right\} \gamma,
\end{aligned}
$$

$$
a_{2}=-\frac{\beta^{2} b^{2}}{2 \alpha(\alpha+2 \beta) c} .
$$

Comparing (20) and $\left(20^{\prime}\right)$ with $\left(12_{1}\right)$ and $\left(11_{1}\right)$, we see that when a point is of type $(\alpha, \alpha, \gamma)$ then

$$
\bar{p}_{x y}=\frac{(\alpha+\gamma)^{2}}{\gamma(2 \alpha+\gamma)} \neq 0,
$$

and therefore 


$$
\begin{aligned}
& \bar{\rho}_{x y}>\rho\left(=\rho_{x y}=a^{2} /(2 b)\right), \quad \text { and } \\
& \bar{\rho}_{x y}\left\{\begin{array}{l}
\geq \\
<
\end{array}\right\} \frac{4}{3} \rho, \text { according as } \gamma\left\{\begin{array}{l}
\equiv \\
>
\end{array}\right\} \alpha .
\end{aligned}
$$

In other words if the radius of curvature $\rho$ of a curve $C$ at a point $O$ is finite and different from zero, then the radius of curvature $\bar{p}_{x y}$ of the section of the tangent surface of $C$ by the osculator at $O$ is always finite and greater than the radius of curvature $\rho_{x y}(=\rho)$ of the projection of $C$ on the osculator, and is greater than, equal to, or less than (4/3) $\rho$, according as the radius of torsion $\tau$ of $C$ at $O$ is zero, finite and different from zero, or infinite. The osculating circle of the section and that of the projection lie on the same side of the tangent.

16. By a similar comparison of (21) and (21') with $\left(14_{1}\right)$ and $\left(15_{1}\right)$, we see that when $\alpha=\beta+\gamma$, then

$$
\bar{\rho}_{x z}=-\frac{\beta^{2}}{\gamma(2 \beta+\gamma)} \rho_{x z} \neq 0 ;
$$

hence $\left|\bar{\rho}_{x z}\right| \neq \rho_{x z}$ and

$$
\left|\boldsymbol{\rho}_{x z}\right|\{\gtrless\} \rho_{x z} \text { according as } \beta\{\gtrless\}\left(1+2^{1 / 2}\right) \gamma
$$

in particular if $\beta=\gamma, \bar{\rho}_{x z}=-\rho_{x z} / 3$.

That is, at a singular point $O$ of type $(\beta+\gamma, \beta, \gamma)$, the radius of curvature $\left|\boldsymbol{\beta}_{x z}\right|$ of the section of the tangent surface by the rectifying plane is never equal to, but is always a rational multiple of, the radius of curvature $\rho_{x z}$ of the projection of the curve on the rectifying plane, and is greater or less than $\rho_{x \varepsilon}$, according as $\beta$ is greater or less than $\left(1+2^{1 / 2}\right) \gamma$. The osculating circle of the section and that of the projection lie on opposite sides of the tangent.

In particular, if the point is of type $(2 \gamma, \gamma, \gamma)$, then $\bar{\rho}_{x z}=-\rho_{x z} / 3$.

Finally, comparing (22) and $\left(22^{\prime}\right)$ with $\left(14_{2}\right)$ and $\left(15_{2}\right)$, we see that if $\alpha+\beta=\gamma$, then

$$
\bar{\rho}_{y z}=-\frac{\beta^{2}}{\alpha(\alpha+2 \beta)} \rho_{y z} \neq 0 ;
$$

hence $\left|\bar{\rho}_{y z}\right| \neq \rho_{y z}$, and

$$
\left|\boldsymbol{\rho}_{y z}\right|\{\gtrless\} \rho_{y z} \text { according as } \beta\{\gtrless\}\left(1+2^{1 / 2}\right) \alpha
$$

in particular, if $\beta=\alpha, \bar{\rho}_{y z}=-\rho_{y z} / 3$.

That is, at a singular point of type $(\alpha, \beta, \alpha+\beta)$ the radius of curvature $\left|\bar{p}_{y z}\right|$ of the section by the normal plane is never equal to, but is always a rational 
multiple of, the radius of curvature $\rho_{y z}$ of the projection on the normal, plane, and is greater or less than $\rho_{y z}$, according as $\beta$ is greater or less than $\left(1+2^{1 / 2}\right) \alpha$. The osculating circle of the section and that of the projection lie on opposite sides of their common tangent (the principal normal of the original curve).

In particular, if the point is of type $(\alpha, \alpha, 2 \alpha)$, then $\bar{\rho}_{y z}=-\rho_{y z} / 3$.

\section{SPHERICAL AND QUASI-SPHERICAL CURVATURE}

17. The dual counterpart of the osculating sphere of a twisted curve $C$ at a point $O$ we shall call its quasi-osculating sphere* at $O$. Its center is the corresponding point on the edge of regression of the rectifying developable and its radius is the distance from that point to the corresponding tangent to $C$. Since the radius $R_{1}$ of the osculating sphere has been called the radius of spherical curvature of $C$, we shall call the radius $R_{2}$ of the quasi-osculating sphere the radius of quasi-spherical curvature of $C$. By means of the known formulas

$$
R_{1}^{2}=\rho^{2}+\left(\frac{\rho^{\prime}}{\eta^{\prime}}\right)^{2}, \quad R_{2}=\left|\frac{s^{\prime}}{\sigma^{\prime}}\right|,
$$

we proceed to find the values of $R_{1}$ and $R_{2}$ at a point $(\alpha, \beta, \gamma)$.

18. Differentiating $\left(8_{1}\right)$ and $\left(8_{3}\right), \S 6$, we obtain

$$
\begin{aligned}
& \rho^{\prime}=\frac{\alpha^{2}(\alpha-\beta) a^{2}}{\beta(\alpha+\beta) b} t^{\alpha-\beta-1}+\cdots, \\
& \sigma^{\prime}=\frac{\alpha \gamma\left(\beta^{2}-\gamma^{2}\right)(\alpha+\beta+\gamma) a c}{\beta^{2}(\alpha+\beta)^{2} b^{2}} t^{\gamma-\beta-1}+\cdots,
\end{aligned}
$$

where the initial coefficients will vanish under the respective conditions $\alpha=\beta, \beta=\gamma$. Some of the succeeding coefficients may also vanish, and we have the following:

$$
\begin{array}{rlrl}
\text { if } & \alpha=\beta, \quad \rho^{\prime}=a_{i} t^{i}+\cdots & & \left(i \geqq 0, a_{i} \neq 0\right) ; \\
\text { if } \quad \beta=\gamma, \quad \sigma^{\prime}=b_{k} t^{k}+\cdots & & \left(k \geqq 0, b_{k} \neq 0\right) .
\end{array}
$$

From $\left(28_{1}\right),\left(29_{1}\right)$ and $\left(9_{3}\right)$ we see that

$$
\begin{aligned}
& \text { if } \alpha \neq \beta, \rho^{\prime} / \eta^{\prime}=\frac{\alpha^{2}(\beta-\alpha) a^{2}}{\gamma(\beta+\gamma)(\alpha+\beta+\gamma) c} t^{\alpha-\beta-\gamma}+\cdots \\
& \text { if } \quad \alpha=\beta, \quad \rho^{\prime} / \eta^{\prime}=-\frac{2 \alpha^{2} b a_{i}}{\gamma(\alpha+\gamma)(2 \alpha+\gamma) c} t^{i+1-\gamma}+\cdots
\end{aligned}
$$

* Quarterly Journal of Mathematics, vol. 46 (1915), pp. 364-366; Mathematische Annalen, vol. 101 (1929), p. 2; G. Loria, Curve Sghembe Speciali, vol. 1, 1921, p. 6; Th. Schmid, Darstellende Geometrie, vol. 1, 1919, p. 164. 
For the sake of brevity we put

$$
\begin{aligned}
& g_{1}=\left|\frac{\alpha^{2}(\beta-\alpha) a^{2}}{\gamma(\beta+\gamma)(\alpha+\beta+\gamma) c}\right|, \\
& h_{1}=\left|\frac{2 \alpha^{2} b a_{i}}{\gamma(\alpha+\gamma)(2 \alpha+\gamma) c}\right|,
\end{aligned}
$$

so that (30) becomes the following:

$$
\begin{array}{ll}
\text { if } \quad \alpha \neq \beta, & \rho^{\prime} / \eta^{\prime}= \pm g_{1} t^{\alpha-\beta-\gamma}+\cdots, \\
\text { if } \quad \alpha=\beta, & \rho^{\prime} / \eta^{\prime}= \pm h_{1} t^{i+1-\gamma}+\cdots .
\end{array}
$$

19. Comparing $\left(27_{1}\right),\left(8_{1}\right)$ and $\left(32_{1}\right)$ we see that if $\alpha \neq \beta$, the significant term in the expression for $R_{1}^{2}$ is $\left(\rho^{\prime} / \eta^{\prime}\right)^{2}$. Hence

$$
\text { if } \alpha \neq \beta, R_{1}=\left|g_{1} t^{\alpha-\beta-\gamma}+\cdots\right| \text {. }
$$

On the other hand if $\alpha=\beta$, so that $\left(8_{1}\right)$ reduces to

$$
\rho=a^{2} /(2 b)+\cdots,
$$

then by comparing $\left(32_{2}\right)$ and (34) we see that the significant term depends on whether $i+1-\gamma$ is negative, zero or positive. Hence

(35) if $\alpha=\beta$ and $\left\{\begin{array}{lll}i+1<\gamma, & \text { then } & R_{1}=\left|h_{1} t^{-(\gamma-t-1)}+\cdots\right|, \\ i+1=\gamma, & \text { then } & R_{1}=\left(h_{1}{ }^{2}+a^{4} /\left(4 b^{2}\right)\right)^{1 / 2}+\cdots, \\ i+1>\gamma, & \text { then } & R_{1}=a^{2} /(2 b)+\cdots\end{array}\right.$

Equations (33) and (35) give the value of $R_{1}$ at a non-singular point in the neighborhood of the singular point $O$. Passing to the limit by letting $t \rightarrow 0$, we find for the value of $R_{1}$ at $O$ the following cases:

(a) If $\alpha>\beta+\gamma, \quad R_{1}=0$.

(b) If $\alpha=\beta+\gamma, \quad R_{1}=g_{1}=a^{2} /(2 c)$.

(c) If $\alpha<\beta+\gamma$ and $\alpha \neq \beta, R_{1}=\infty$.

(d) If $\alpha=\beta$ and $i+1>\gamma, R_{1}=a^{2} /(2 b)$.

(e) If $\alpha=\beta$ and $i+1=\gamma, \quad R_{1}=\left(h_{1}^{2}+a^{4} /\left(4 b^{2}\right)\right)^{1 / 2}$.

(f) If $\alpha=\beta$ and $i+1<\gamma, R_{1}=\infty$.

In cases (b), (d) and (e), in view of (31), $R_{1}$ is finite and $\neq 0$. If $\gamma=1$, case (f) does not occur. 
20. Turning now to $R_{2}$, given by $\left(27_{2}\right)$, we soon find that by using $\left(9_{1}\right)$, $\left(28_{2}\right)$ and $\left(29_{2}\right)$ and putting

$$
\begin{aligned}
& g_{2}=\left|\frac{\beta^{2}(\alpha+\beta)^{2} b^{2}}{\gamma\left(\beta^{2}-\gamma^{2}\right)(\alpha+\beta+\gamma) c}\right|, \\
& h_{2}=\left|\frac{\alpha a}{b_{k}}\right|,
\end{aligned}
$$

we obtain the following expansions of $R_{2}$ :

$$
\begin{array}{lll}
\text { if } \beta \neq \gamma, & R_{2}=\left|g_{2} t^{\alpha+\beta-\gamma}+\cdots\right| ; \\
\text { if } \beta=\gamma, & R_{2}=\left|h_{2} t^{\alpha-k-1}+\cdots\right| .
\end{array}
$$

Again passing to the limit as $t \rightarrow 0$, we find for the value of $R_{2}$ at the singular point $O$ the following cases:

$$
\begin{aligned}
& \text { (a) If } \alpha+\beta<\gamma, R_{2}=\infty . \\
& \text { (b) If } \alpha+\beta=\gamma, \quad R_{2}=g_{2}=\beta^{2} b^{2} /(2 \alpha(\alpha+2 \beta) c) \text {. } \\
& \text { (c) If } \alpha+\beta>\gamma, \quad \text { and } \beta \neq \gamma, \quad R_{2}=0 . \\
& \text { (d) If } \beta=\gamma \text { and } k+1>\alpha, \quad R_{2}=\infty . \\
& \text { (e) If } \beta=\gamma \text { and } k+1=\alpha, \quad R_{2}=h_{2} . \\
& \text { (f) If } \beta=\gamma \text { and } k+1<\alpha, \quad R_{2}=0 .
\end{aligned}
$$

In cases (b) and (e), $R_{2}$ is finite and $\neq 0$. If $\alpha=1$, case (f) does not occur. 21. From (36) and (39) we easily derive a number of interesting consequences. In view of (14), (21), (22) and (12) we see that $R_{1}$ is closely connected with $\rho_{x z}, \bar{\rho}_{x z}$ and $\rho$, and that $R_{2}$ is connected with $\rho_{y z}, \bar{\rho}_{y z}$ and $\sigma$. Indeed:

The radius of spherical curvature $R_{1}$ at a singular point $O$ is zero, infinite or neither, according as the radii of curvature $\rho_{x z}$ and $\bar{\rho}_{x z}$ (of the projection on, and the section by, the rectifying plane at $O$ ) are zero, infinite or neither, provided that when $\rho_{x z}$ and $\bar{\rho}_{x z}$ are infinite, the polar line at $O$ either coincides with the binormal or lies at infinity; $\rho_{x z}$ and $\bar{p}_{x z}$ are zero, if, and only if, $R_{1}$ is zero.

If $\rho$ is finite and $\neq 0$ and if the osculator is simple $(\gamma=1)$, then $R_{1}$ is finite and $\neq 0$.
The radius of quasi-spherical curvature $R_{2}$ at a singular point $O$ is zero, infinite or neither, according as the radii of curvature $\rho_{y z}$ and $\bar{\rho}_{y z}$ (of the projection on, and the section by, the normal plane at $O$ ) are zero, infinite or neither, provided that when $\rho_{y z}$ and $\bar{\rho}_{y z}$ are zero, the rectifying line at $O$ coincides either with the binormal or with the tangent; $\rho_{y z}$ and $\bar{\rho}_{y z}$ are infinite, if and only if $R_{2}$ is infinite and $\sigma$ is zero.

If $\sigma$ is finite and $\neq 0$ and if the point is simple $(\alpha=1)$, then $R_{2}$ cannot vanish. 
If $\rho_{x z}$ and $\bar{\rho}_{x z}$ are finite and $\neq 0$ $(\alpha=\beta+\gamma)$, we can say more. For by comparing (36), case (b), with (14 and $\left(15_{1}\right)$ we see that $R_{1}$ is not only finite and $\neq 0$, but is actually equal to $\rho_{x z}$; also since $\rho=0(\alpha>\beta)$, the polar line coincides with the binormal.

Hence if the curvature of the projection of the curve on the rectifying plane is finite and different from zero $(\alpha=\beta+\gamma)$, then the osculating circle of this projection is a great circle of the corresponding osculating sphere of the original curve. This sphere will lie on the positive side $(z>0)$ of the osculator.
If $\rho_{y z}$ and $\bar{\rho}_{y z}$ are finite and $\neq 0$ $(\alpha+\beta=\gamma)$, we can say more. For by comparing (39), case (b), with (22) and $\left(22^{\prime}\right)$ we see that $R_{2}$ is not only finite and $\neq 0$, but is actually equal to $\left|\bar{\rho}_{y_{2}}\right|$; also since $\sigma=0(\gamma>\beta)$, the rectifying line coincides with the binormal.

If the curvature of the section of the tangent surface by the normal plane is finite and different from zero $(\alpha+\beta$ $=\gamma$ ), then the osculating circle of this section is a great circle of the corresponding quasi-osculating sphere of the original curve. This sphere will lie on the negative side $(z<0)$ of the osculator.

22. If $\alpha=\beta+\gamma$, either all three of the integers $\alpha, \beta, \gamma$ are even or just one is even, so that by $\S 4$ the point belongs to category $1,2,3$ or 4 . Hence, by $\$ \$ 12$ and 15 , we see that if the negative branch of the curve lies in one of the octants $5,6,7$ or 8 ,

and if $\rho=0(\alpha>\beta)$, then $R_{1}, \rho_{x z}, \quad$ and if $\sigma=0(\gamma>\beta)$, then $R_{2}, \rho_{x z}, \bar{\rho}_{x z}$, $\bar{\rho}_{x z}, \rho_{y z}$, and $\bar{\rho}_{y z}$ are all $=0$ or $\infty$. $\rho_{y z}$ and $\bar{\rho}_{y z}$ are all $=0$ or $\infty$.

In other words if the two branches of the curve at a point $O$ lie on opposite sides of the osculating plane,

and if the polar line at $O$ coincides with the binormal, then no one of the five variables $\rho_{x z}, \bar{\rho}_{x z}, \rho_{y z}, \bar{\rho}_{y z}, R_{1}$ can be finite and different from zero.

If $R_{1}$ is finite and $\neq 0$, either $\alpha=\beta+\gamma$ or $\alpha=\beta$. By $\S 8$ this can happen only for points of categories $1,2,3,4,7$ or 8 .

Hence if the negative branch of the curve lies in the 5th or 6th octant, no one of the four variables $\rho, \rho_{x z}, \bar{\rho}_{x z}, R_{1}$ can be finite and different from zero. and if the rectifying line at $O$ coincides with the binormal, then no one of the five variables $\rho_{x z}, \bar{\rho}_{x z}, \rho_{y z}, \bar{\rho}_{y z}, R_{2}$ can be finite and different from zero.

If $R_{2}$ is finite and $\neq 0$, either $\alpha+\beta=\gamma$ or $\beta=\gamma$. By $\S 8$ this can happen only for points of categories $1,2,3,4,5$ or 8 .

Hence if the negative branch of the curve lies in the 6th or 7th octant, no one of the four variables $\sigma, \rho_{y z}, \bar{\rho}_{y z}, R_{2}$ can be finite and different from zero. 
An immediate consequence is the remarkable fact that if the negative branch lies in the 6 th octant $[+1,-1,-1]$, no one of the eight variables $\rho$, $\sigma, \rho_{x z}, \bar{\rho}_{x z}, \rho_{y z}, \bar{\rho}_{y z}, R_{1}, R_{2}$ can be finite and different from zero. The simplest example of this is a point of type $(2,1,2)$.

23. Finally we shall exhibit in the following table some of our results as applied to a number of the simpler types of singular points, together with the type $(1,1,1)$ of non-singular points. Here $a, b, c, c_{1}$, and $h_{2}$ are all finite and $\neq 0 ; a, b, c$ are the coefficients in the equations (5) of $\S 3 ; h_{2}$ is given by $\left(37_{2}\right)$ of $\$ 20$; and $c_{1}$ is the value of $R_{1}$ when it may come under case (d) or case (e) of (36), $\$ 19$.

The table includes the twenty-seven types for which $\alpha, \beta, \gamma$ are $\leqq 3$ and a few others. Wherever three types are grouped together, the first and third are dual and the second is self-dual. All of the types listed belong to distinct species except $(1,1,1)$ and $(3,3,3)$; the latter is the simplest type of singular point that looks exactly like a non-singular point.

\begin{tabular}{|c|c|c|c|c|c|c|c|c|c|c|c|c|c|}
\hline & $\alpha \beta \gamma$ & $\begin{array}{l}\alpha, \alpha+\beta \\
\alpha+\beta+\gamma\end{array}$ & $\begin{array}{l}\text { Oct- } \\
\text { ant }\end{array}$ & $\rho$ & $\tau$ & $\sigma$ & $\rho_{x z}$ & $\bar{\rho}_{x z}$ & $R_{1}$ & $\bar{\rho}_{x y}$ & $\rho_{y z}$ & $\bar{\rho}_{y z}$ & $R_{2}$ \\
\hline 1 & 111 & 123 & 8 & $\frac{a^{2}}{2 b}$ & $-\frac{a b}{3 c}$ & $-\frac{3 a c}{2 b^{2}}$ & $\infty$ & $\infty$ & $c_{1}$ & $\frac{2 a^{2}}{3 b}$ & 0 & 0 & $h_{2}$ or $\infty$ \\
\hline 2 & 112 & 124 & 2 & $\overline{\frac{a^{2}}{2 b}}$ & $\infty$ & 0 & $\infty$ & $\infty$ & $c_{1}$ or $\infty$ & $\frac{9 a^{2}}{16 b}$ & $\frac{b^{2}}{2 c}$ & $-\frac{b^{2}}{6 c}$ & $\frac{b^{2}}{6 c}$ \\
\hline 3 & 121 & 134 & 3 & $\infty$ & $-\frac{a b}{2 c}$ & & $\infty$ & $\infty$ & $\infty$ & $\infty$ & 0 & 0 & \\
\hline 4 & 211 & 234 & 4 & 0 & 0 & $-\frac{16 a c}{9 b^{2}}$ & $\frac{a^{2}}{2 c}$ & $-\frac{a^{2}}{6 c}$ & $\frac{a^{2}}{2 c}$ & 0 & 0 & 0 & $0, h_{2}$ or $\infty$ \\
\hline 5 & 122 & 136 & 5 & $\infty$ & $\infty$ & $-\frac{10 a c}{9 b^{2}}$ & $\infty$ & $\infty$ & $\infty$ & $\infty$ & 0 & 0 & $h_{2}$ or $\infty$ \\
\hline 6 & 212 & 235 & 6 & 0 & $-\frac{a b}{5 c}$ & 0 & $\infty$ & $\infty$ & $\infty$ & 0 & 0 & 0 & 0 \\
\hline 7 & 221 & 245 & 7 & $\frac{a^{2}}{2 b}$ & 0 & $\infty$ & $\infty$ & $\infty$ & $c_{1}$ & $\frac{9 a^{2}}{10 b}$ & 0 & 0 & 0 \\
\hline 8 & 222 & 246 & 1 & $\frac{a^{2}}{2 b}$ & $-\frac{a b}{3 c}$ & $-\frac{3 a c}{2 b^{2}}$ & $\infty$ & $\infty$ & $c_{1}$ or $\infty$ & $\frac{2 a^{2}}{3 b}$ & 0 & 0 & $0, h_{2}$ or $\infty$ \\
\hline 9 & 123 & 136 & 3 & $\infty$ & $\infty$ & 0 & $\infty$ & $\infty$ & $\infty$ & $\infty$ & $\frac{b^{2}}{2 c}$ & $-\frac{2 b^{2}}{5 c}$ & $\frac{2 b^{2}}{5 c}$ \\
\hline 10 & 132 & 146 & 2 & $\infty$ & $\infty$ & $\infty$ & $\infty$ & $\infty$ & $\infty$ & $\infty$ & 0 & 0 & 0 \\
\hline 11 & 213 & 236 & 4 & 0 & $\infty$ & 0 & $\infty$ & $\infty$ & $\infty$ & 0 & $\frac{b^{2}}{2 c}$ & $-\frac{b^{2}}{16 c}$ & $\frac{b^{2}}{16 c}$ \\
\hline 12 & 231 & 256 & 4 & $\infty$ & 0 & $\infty$ & $\infty$ & $\infty$ & $\infty$ & $\infty$ & 0 & 0 & 0 \\
\hline 13 & 312 & 346 & 2 & 0 & 0 & 0 & $\frac{a^{2}}{2 c}$ & $-\frac{a^{2}}{16 c}$ & $\frac{a^{2}}{2 c}$ & 0 & 0 & 0 & 0 \\
\hline 14 & 321 & 356 & 3 & 0 & 0 & $\infty$ & $\frac{a^{2}}{2 c}$ & $-\frac{2 a^{2}}{5 c}$ & $\frac{a^{2}}{2 c}$ & 0 & 0 & 0 & 0 \\
\hline
\end{tabular}




\begin{tabular}{|c|c|c|c|c|c|c|c|c|c|c|c|c|c|}
\hline & $\alpha \beta \gamma$ & $\mid \begin{array}{l}\alpha, \alpha+\beta \\
\alpha+\beta+\gamma\end{array}$ & $\begin{array}{r}\text { Oct- } \\
\text { ant }\end{array}$ & $\rho$ & $\tau$ & $\sigma$ & $\rho_{x z}$ & $\rho_{x z}$ & $R_{1}$ & $\rho_{x z}$ & $\rho_{y z}$ & $\rho_{y z}$ & $R$, \\
\hline 15 & 113 & 125 & 8 & $\frac{a^{2}}{2 b}$ & $\infty$ & 0 & $\infty$ & $\infty$ & $c_{1}$ or $\infty$ & $\frac{8 a^{2}}{15 b}$ & $\infty$ & $\infty$ & $\infty$ \\
\hline 16 & 131 & 145 & 8 & $\infty$ & $-\frac{3 a b}{5 c}$ & $\infty$ & $\infty$ & $\infty$ & $\infty$ & $\infty$ & 0 & 0 & \\
\hline 17 & 311 & 345 & 8 & 0 & 0 & $-\frac{15 a c}{8 b^{2}}$ & 0 & 0 & 0 & 0 & 0 & 0 & $0, h_{2}$ or $\infty$ \\
\hline 18 & 133 & 147 & 8 & $\infty$ & $\infty$ & $-\frac{7 a c}{8 b^{2}}$ & $\infty$ & $\infty$ & $\infty$ & $\infty$ & 0 & 0 & $h_{2}$ or $\infty$ \\
\hline 19 & 313 & 347 & 8 & 0 & $-\frac{a b}{7 c}$ & 0 & $\infty$ & $\infty$ & $\infty$ & 0 & 0 & 0 & 0 \\
\hline 20 & 331 & 367 & 8 & $\frac{a^{\circ}}{2 b}$ & 0 & $\infty$ & $\infty$ & $\infty$ & $c_{1}$ & $\frac{8 a^{a}}{7 b}$ & 0 & 0 & 0 \\
\hline 21 & 223 & 247 & 7 & $\frac{a^{2}}{2 b}$ & $\infty$ & 0 & $\infty$ & $\infty$ & $c_{1}$ or $\infty$ & $\frac{25 a^{2}}{42 b}$ & 0 & 0 & 0 \\
\hline 22 & 232 & 257 & 6 & $\infty$ & $-\frac{5 a v}{7 c}$ & $\infty$ & $\infty$ & $\infty$ & $\infty$ & $\infty$ & 0 & 0 & \\
\hline 23 & 322 & 357 & 5 & 0 & 0 & $-\frac{42 a c}{25 b^{2}}$ & $\infty$ & $\infty$ & $\infty$ & 0 & 0 & 0 & $0, h_{2}$ or $\infty$ \\
\hline 24 & 233 & 258 & 4 & $\infty$ & $\infty$ & $-\frac{32 a c}{25 b^{2}}$ & $\infty$ & $\infty$ & $\infty$ & $\infty$ & 0 & 0 & $0, h_{3}$ or $\infty$ \\
\hline 25 & 323 & 358 & 3 & 0 & $-\frac{a 0}{4 c}$ & 0 & $\infty$ & $\infty$ & $\infty$ & $\begin{array}{c}0 \\
25 a^{2}\end{array}$ & 0 & 0 & $\mathbf{0}$ \\
\hline 26 & 332 & 368 & 2 & $\frac{a}{2 b}$ & & $\infty$ & $\infty$ & $\infty$ & $c_{1}$ or $\infty$ & $\frac{20 b}{32 b}$ & 0 & 0 & 0 \\
\hline 27 & 333 & 369 & 8 & $\frac{a^{2}}{2 b}$ & $-\frac{a b}{3 c}$ & $-\frac{3 a c}{2 b^{2}}$ & $\infty$ & $\infty$ & $c_{1}$ or $\infty$ & $\frac{2 a^{2}}{3 b}$ & 0 & 0 & $0, h_{2}$ or $\infty$ \\
\hline 28 & 124 & 137 & $J$ & $\infty$ & $\infty$ & 0 & $\infty$ & $\infty$ & $\infty$ & $\infty$ & $\infty$ & $\infty$ & $\infty$ \\
\hline 29 & 142 & 157 & 5 & $\infty$ & $\infty$ & $\infty$ & $\infty$ & $\infty$ & $\infty$ & $\infty$ & 0 & 0 & 0 \\
\hline 30 & 214 & 237 & 6 & 0 & $\infty$ & 0 & $\infty$ & $\infty$ & $\infty$ & 0 & $\infty$ & $\infty$ & $\infty$ \\
\hline 31 & .241 & 267 & 7 & $\infty$ & 0 & $\infty$ & $\infty$ & $\infty$ & $\infty$ & $\infty$ & 0 & 0 & 0 \\
\hline 32 & 412 & 457 & 6 & 0 & 0 & 0 & 0 & 0 & 0 & 0 & 0 & 0 & 0 \\
\hline 33 & 421 & 467 & 7 & 0 & 0 & $\infty$ & 0 & 0 & 0 & 0 & 0 & 0 & 0 \\
\hline 34 & 224 & 248 & 1 & $\frac{a^{2}}{2 b}$ & $\infty$ & 0 & $\infty$ & $\infty$ & $c_{1}$ or $\infty$ & $\overline{\frac{9 a^{2}}{16 b}}$ & $\frac{b^{2}}{2 c}$ & $-\frac{b^{2}}{6 c}$ & $\frac{b^{2}}{6 c}$ \\
\hline 35 & 242 & 268 & 1 & $\infty$ & $-\frac{a v}{2 c}$ & $\infty$ & $\infty$ & $\infty$ & $\infty$ & $\infty$ & 0 & 0 & 0 \\
\hline 36 & 422 & 468 & 1 & 0 & 0 & $-\frac{10 a c}{9 b^{2}}$ & $\frac{a^{*}}{2 c}$ & $-\frac{a^{2}}{6 c}$ & $\frac{a^{2}}{2 c}$ & 0 & 0 & 0 & $0, h_{2}$ or $\infty$ \\
\hline
\end{tabular}

Cornell University,

ITHACA, $\mathbf{N}$. $\mathbf{Y}$. 